Discussion Paper No. 726

\title{
TIME DISCOUNTING: \\ THE DELAY EFFECT AND \\ PROCRASTINATING BEHAVIOR
}

\author{
Shunichiro Sasaki, Shiyu Xie, \\ Shinsuke Ikeda, Jie Qin and Yoshiro Tsutsui
}

December 2008

The Institute of Social and Economic Research Osaka University

6-1 Mihogaoka, Ibaraki, Osaka 567-0047, Japan 


\title{
Time Discounting: The Delay Effect and Procrastinating Behavior ${ }^{*}$
}

\author{
Shunichiro Sasaki ${ }^{\mathrm{a}}$ \\ (Nagoya University of Commerce and Business) \\ Shiyu Xie ${ }^{\mathrm{b}}$ \\ (Fudan University) \\ Shinsuke Ikeda ${ }^{\mathrm{c}}$ \\ (Oasaka University) \\ Jie Qin ${ }^{\mathrm{d}}$ \\ (Ritsumeikan University) \\ Yoshiro Tsutsui ${ }^{\mathrm{e}}$ \\ (Osaka University)
}

\footnotetext{
* We are grateful to Fumio Ohtake, Junmin Wan, and Sun Bibo for their support and comments. This research was financially supported by the 21 st Century COE program at Osaka University and Grant-in-Aid for Scientific Research No. 17203025 of the Ministry of Education, Culture, Sports, Science and Technology in Japan.

${ }^{a}$ Faculty of Economics, Nagoya University of Commerce and Business, 4-4 Sagamine, Komenoki-cho, Nisshin, Aichi 470-0193, Japan; e-mail: ssasaki@nucba.ac.jp, Phone: +81-561-73-2111, Fax: +81-561-73-1202

b Department of World Economics, Fudan University, 220 Handan Road, Shanghai 200433, P. R. China; e-mail: syxie@fudan.edu.cn

c Institute of Social and Economic Research, Osaka University, 6-1 Mihogaoka, Ibaraki, Osaka 567-0047, Japan; e-mail: ikeda@iser.osaka-u.ac.jp

d Faculty of Economics, Ritsumeikan University, 1-1-1 Noji-higashi, Kusatsu, Shiga 525-8577, Japan; e-mail: jieqinjp@yahoo.co.jp

e Corresponding author, Graduate School of Economics, Osaka University, 1-7 Machikaneyama, Toyonaka, 560-0043 Japan, e-mail: tsutsui@econ.osaka-u.ac.jp
} 


\begin{abstract}
The delay effect, that people discount the near future more than the distant future, has not been verified rigorously. An experiment conducted by us in China confirms that, by separating the delay from the interval, the delay effect exists only within a short delay. The results are reliable, because the rewards paid were very large, in order to elicit the subjects' true preferences. The interval and magnitude effects are also confirmed. Finally, subjects' procrastinating behavior, as reported in the questionnaire conducted at the end of the experiment, is explained by the time discount rates and the degree of the delay effect revealed in the experiment.
\end{abstract}

JEL classification numbers: C91; D91

Keywords: discount rate; economic experiment in China; delay effect; naïfs and sophisticates; procrastination 


\section{Introduction}

This study examines an economic experiment conducted in China to explore how people discount future values. Although traditional economics has relied on the assumption of "exponential discounting," i.e., that the time discount rate is constant over the time horizon, many experimental studies have found that the opposite is true: that is, people do not discount future values exponentially (Ainslie 1975, Benzion et al. 1989, Kirby 1997, Kirby and Marakovic 1995, and Thaler 1981). This anomaly of discounting, that it is not constant over the time horizon, is called the delay effect, diminishing impatience, or hyperbolic discounting. In addition, it is known that discount rates depend on the value to be discounted: the smaller the value, the more intensively it is discounted, and this is called "the magnitude effect" (Benzion et al. 1989, Kirby and Marakovic 1995, Kirby 1997, and Thaler 1981).

However, most studies of the delay effect face a problem. In experiments thus far, subjects are typically asked which of two options is more preferable: option A, in which the subjects will receive a reward in the near future, or option $\mathrm{B}$, in which the subjects will receive a (usually larger) reward in the distant future. All options shown in the question are neatly identified with four experimental conditions: date $s$ and amount $X$ of receipt in option A; and date $t(t>s)$ and amount $Y$ (usually larger than $X$ ) in option B. Associated with these conditions, we call $s$ the delay and the difference between the two points of time, $t-s$, the interval $h$. In what follows, we call the phenomenon that discount rates depend on the delay "the delay effect," on the interval "the interval effect," and on the reward amount "the magnitude effect."

Thus, the two concepts of time, the delay $s$ and the interval $h$, should be distinguished as the factors that characterize intertemporal choices. The delay $s$ is, 
among other things, the focal point, because if time discounting depends on the delay, a plan made by such a person will be time-inconsistent. Nonetheless, most studies so far have not separated the delay from the interval, and subjects are asked to specify the amount of money that they would require, at some future date, to make them indifferent to receiving some amount now. In these studies, since the receipt time of the earlier option is fixed at now, their results should be interpreted as an investigation of the interval effect rather than the delay effect or, at least, as a mixture of these two effects.

Read (2001) was the first to point out this problem. He conducted an experiment that separated the delay from the interval. He observed a form of the interval effect, but did not confirm the delay effect. ${ }^{1}$ However, although the delay effect exists even with quite a short delay (Frederick et al. 2002), the shortest delay used by Read was six months. Thus, whether subjects show the delay effect within a six-month delay remains an open question. Given these arguments, the primary aim of this study is to reexamine whether the delay effect is in fact found when the delay and the interval are explicitly distinguished. Our experiment differs from Read (2001) in that we investigated the delay effect using a much shorter delay than his.

The second aim is to explain the procrastinating behavior of the subjects by their time discount rates and the degree of the delay effect. Although the delay effect is expected to cause procrastinating behaviors, because it means a high discount rate in the near future, it subsequently makes people regret that they procrastinated. Therefore,

\footnotetext{
${ }^{1}$ Recently, Read and Roelofsma (2003) and Read et al. (2005) have reported that the delay effect is found in experiments in which subjects are asked questions in ways that differ from those in Read (2001). Read and Roelofsma (2003) utilized the matching method, which asks subjects about the amount at a specified date, which makes them indifferent to a specified option. This is different from the choice method used in Read (2001) and in the current study, which asks subjects to choose the better of two specified options. On the other hand, Read et al. (2005) focused on how the timing of two options is described and specified the timing with calendar dates. This method is different from Read (2001) and from the current paper, which specified the length of the delay.
} 
after repeating this serious regret, they would stop procrastinating. Thus, the relation between the delay effect and procrastinating behavior is not straightforward. In this study, we investigate the relation based on the concept of naïfs and sophisticates, as developed by O’Donoghue and Rabin (1999).

One merit of our study is that, in our experiments, we give the Chinese subjects strong incentives, by offering a high reward to induce them to reveal their true preference. This study examines whether real payment to all subjects is indispensable for eliciting the true preference when some subjects are really paid a large reward.

The remainder of this paper is organized as follows. In the next section, we explain our experimental design. In section 3, we report the results of the experiment and examine whether the delay, the interval, and the magnitude effects are confirmed by means of the test of the same mean, and a regression analysis. In section 4 , we examine whether the discount rate and the degree of the delay effect of the subjects can explain their procrastinating behavior. In section 5, we present our conclusion.

\section{Design of the experiment}

We try to measure subjective time discounting by asking subjects which of the two options they prefer: option A, to receive a reward $X$ with a shorter delay $s$, and option B, to receive a (usually larger) reward $Y$, with a longer delay $t$. From these conditions, the

rate of compensation for a postponement $R$ is defined as $\frac{1}{X} \frac{Y-X}{t-s}$, and the interval $h$ is defined as $t-s$.

We specified four values for $s$ ( 2 days, 1 month, 90 days, 10 months), three values for $h$ (7 days, 3 months, 12 months), and three values for the reward amount (240, 2800, and 800000 yuan). We also controlled for whether the reward specified in the pay-off 
tables would really be paid, which we denote as $P=($ YES, NO). Combining these four conditions, we specified twelve sets of conditions (s, $h, X, P)$, as shown in Table 1 . Thus, the experiment consists of twelve rounds, each of which corresponds to one set of conditions.

For each set of conditions, we asked about 32 pairs of options, each of which is specified with a value of $R$ such that the value of the reward of the later option is $Y$. Thirty-two pairs of options are arranged in ascending order of $R$, which varies from a very low value of $-20 \%$ to a very high value of $300 \%$ (annual rate), in a sheet that we call the "payoff table." 2 The subjects are expected to choose the earlier receipt A when $R$ is small and the later receipt B when $R$ becomes sufficiently large. Then, we define the time discount rate $T D_{\mathrm{i}, \mathrm{j}}$ for the condition $j=(s, h, X, P)$ and the subject $i$ with the value of $R$ at which the subject switched from $\mathrm{A}$ to $\mathrm{B} .^{3}$

The experiment was conducted on March 11, 2005 at Fudan University, Shanghai. ${ }^{4}$ The subjects were 29 undergraduate students recruited from the Department of World Economics at Fudan University. They were either 20 or 21 years old, and 25 of the 29 subjects $(86 \%)$ were female. The reward was paid by the following procedure. At the end of the experiment, starting from round one, we randomly selected one pair of options to be paid from the payoff table. Then we randomly selected one subject as a recipient of the reward amount on the date specified in the option that the subject had chosen. $^{5}$ The highest reward presented in the payoff tables was 3920 yuan $(800000$

\footnotetext{
2 One payoff table is presented in Appendix.

${ }^{3}$ If a subject chooses A (B) for all 32 pairs, we regard the highest (lowest) value of $R$ in the payoff table as $T D_{\mathrm{ij}}$. If a subject switches multiple times, we discard the data because the subject had probably misunderstood the question.

${ }^{4}$ We conducted an experiment on risk aversion before the experiment on the time discount rate, on the same day. For the experiment on risk aversion, see Sasaki et al. (2008).

${ }^{5}$ Since this payment procedure was explained in the instructions and was rehearsed twice, the
} 
yuan would not be paid), which was a relatively large amount, considering that the annual household income of the subjects was $20-40$ thousand yuan. This helped to give the subjects an incentive to express their true preferences. Including a 120 yuan (about US\$14) participation fee, the average payoff for both the experiments, on time discounting and risk aversion, was 788 yuan (about US\$106). After we selected the winners, the subjects were required to complete a detailed questionnaire requesting their preferences, opinions, and attributes.

\section{Results of the experiment}

\subsection{Time discount rates elicited by the experiment}

In the bottom row of Table 1, we present the average and standard error of the discount rates, which reveal that the discount rates are distributed over a wide range from $1.0 \%$ to $44 \%$ (in the annual rate), depending on the experimental conditions. This implies that the conventional assumption of exponential discounting is not supported by the data. In other words, there exist anomalies that are probably associated with the delay, interval, and reward amount. In this section, we examine the delay, interval, and magnitude effects. We also investigate whether the results depend on the promise of the payment of a reward.

3.2 Examination of the three anomalies in time discounting by means of tests of the same mean

In our framework, the discount rates may differ depending on four experimental conditions: the delay $s$, the interval $h$, the reward amount $X$, and whether the payment is

subjects were expected to understand clearly that what they received would depend on their choice. 
promised $P$. In this subsection, in each round we hold three of the four conditions constant and vary the fourth condition, and we test whether the mean discount rate varies.

\section{The delay effect}

To test the delay effect, we compare the rounds in which the interval and amount are the same but the delay differs. ${ }^{6}$ They are as follows:

1) Round 2 (30 days, 90 days, 240 yuan) vs. Round 4 (10 months, 90 days, 240 yuan)

2) Round 5 ( 2 days, 7 days, 2800 yuan) vs. Round 6 (90 days, 7 days, 2800 yuan)

3) Round 7 (1 month, 3 months, 2800 yuan) vs. Round 9 (10 months, 3 months, 2800 yuan)

4) Round 10 (1 month, 3 months, 800000 yuan) vs. Round 12 (10 months, 3 months, 800000 yuan)

The results of the test of the same mean are presented in Table 2 . In all the combinations, the null hypothesis of the same mean is not rejected. Thus, the delay effect is not observed by the test of the same mean.

\section{The interval effect}

For the interval effect, three comparison tests, round 2 vs. 3 , round 7 vs. 8 , and round 10 vs. 11, are possible, and the test results are presented in Table 3 . When the reward amount is set at 240 or 2800 yuan, the discount rates are significantly higher in the shorter intervals than in the longer intervals, and this confirms the interval effect.

\footnotetext{
${ }^{6}$ Whether the payment would be made or not is also controlled.
} 
However, when the reward amount is set at 800000 yuan, the null of the same mean is not rejected. This is probably because people will choose sufficiently low discount rates for an extremely large amount such as 800000 yuan, so that a difference in the other conditions no longer changes the discount rates. The result of the interval effect is generally consistent with the subadditivity found by Read (2001).

\section{The magnitude effect}

The results of the tests of the magnitude effect are presented in Table 4. In the top panel, we show three test results comparing the reward of 240 and 2800 yuan. Results comparing the reward of 2800 and 800000 yuan are presented in the middle panel, and those comparing the reward of 240 and 800000 yuan are presented in the bottom panel. In all the combinations, the time discount rate is significantly higher for the smaller reward amount. Thus, the magnitude effect, in which the discount rates are inversely related to the reward amount, is significantly observed. This is consistent with the results of previous studies (Benzion et al. 1989, Kirby 1997, Kirby and Marakovic 1997, and Thaler 1981).

\section{The payment effect}

Finally, we examine whether the promise of payment affects the discount rates. Rounds 1 and 7 are designed to have the same condition $(s, h, X)$, and they differ only in that the reward is actually paid in round 5 but not in round 1 . The test result of the same mean is presented in Table 5, which reveals that the null of the same mean is not rejected at all. As noted above, the reward of 2800 yuan was relatively large in China, and this result therefore has a strong implication: whether the reward is actually paid to the subjects has nothing to do with the time discount rates they report, as long as some of the 
subjects will really be paid a large reward. This provides grounds for the validity of our experimental result with respect to the following: 1) although we did not pay rewards to all subjects except the winner of each round, the results as a whole are trustworthy, and 2 ) the results of rounds $10-12$, where the reward of 80000 yuan is only imaginary, are also reliable.

\subsection{Regression analysis for an investigation of three anomalies}

In the last subsection, we did not confirm the delay effect, but we did find the interval and magnitude effects. This is consistent with Read's (2001) conclusion, and it implies that the conclusion of many previous studies that argued for the delay effect is false, because they did not separate the delay from the interval. However, we should not be hasty, because the comparison tests in the previous subsection may suffer from the problem of a small sample, and this may lead to a small power to reject the null of the same mean. Looking at Table 2 carefully, we see that the rounds of the 2-days delay reports a discount rate of $30.6 \%$, which is substantially higher than $22 \%$ in round 6 , for the 90-days delay. Although the p-value is only $32 \%$, this may be a result of the small sample of 29. To avoid this weakness, we pool all the samples of 348 , and conduct a regression analysis using them.

The regression equation basically controlled all the experimental conditions: the delay $s$, the interval $h$, the reward amount $X$, and whether the reward is actually paid $P$. In particular, we adopt the following variables. For the variables standing for the delay, we use dummy variables $H O R 2_{j}, H O R 30_{j}$, and $H O R 90_{j}$, which is equal to unity if the delay is 2 days, 1 month, and 3 months in round $j$, respectively, and 0 otherwise (10 
months). For the amount of the reward, we use dummy variables $A M T 240_{j}$ and $A M T 2800_{j}$, which is equal to unity if the amount of reward is 240 and 2800 yuan, respectively, in round $j$, and 0 otherwise (800000 yuan). For the interval, we use a variable $I N T_{j}$, which is equal to the length of the interval in round $j$. Finally, we use a dummy variable $P A Y_{j}$, which is equal to unity if the reward will be paid in round $j$, and 0 otherwise. Thus, the regression equation is

$$
\begin{aligned}
& \ln T D_{i j}=\alpha_{0}+\alpha_{1} \mathrm{HOR}_{j}+\alpha_{2} \mathrm{HOR}_{3} 0_{j}+\alpha_{3} \mathrm{HOR}_{j}+\alpha_{4} \mathrm{INT} \mathrm{T}_{j}+\alpha_{5} \mathrm{AMT}_{2} 40_{j} \\
& +\alpha_{6} A M T 2800_{j}+\alpha_{7} P A Y_{j}+\varepsilon_{i, j}
\end{aligned}
$$

where $\ln T D_{i, j}$ stands for the logarithm of subject i's time discount rate in round $j$.

The results of the regression are presented in Table 6. For the variables of the delay dummies, the estimated coefficient of $H O R 2_{j}$ is significantly positive, but those of $\mathrm{HOR} 30_{j}$ and $\mathrm{HOR} 90_{j}$ are insignificant, indicating that the delay effect operates only in 2-days delay, but not in the rounds where the delay is more than 30 days. This non-linear property of the delay effect is close to the quasi-hyperbolic discount model, in that people discount much of the future value between the present and the very near future, whereas they discount it moderately after that period (Laibson 1997). The result does not contradict Read (2001), who reported that the delay effect is not observed in the case of 6-months and 8-months delays, which are both substantially longer than ours.

Let us briefly examine the other anomalies. The estimated coefficients of $A M T 240_{j}$ and $A M T 2800_{j}$ are significantly positive, and that of $I N T_{j}$ is significantly negative. These results confirm the magnitude and interval effects, as we have already 
confirmed by the test of the same mean. The estimated coefficient of $P A Y_{j}$ is insignificant, which is also consistent with the results in the previous subsection. Thus, when a part of the subjects are randomly chosen, and are actually paid a large reward amount, the subjects answer honestly those questions in which the reward is imaginary. Therefore, all of their responses in our experiment are reliable.

Next, we consider the attributes of the subjects, gender, and household income. In particular, we add a dummy variable, $M A L E$, which is equal to unity for male subjects and zero otherwise, and a dummy variable HINCOME, which is equal to unity if the subject's annual household income is over 140 thousand yuan and zero otherwise. Five subjects are classified as high income and 18 as low income. Since 7 subjects who did not report household income were excluded, the number of observations is 23 for this regression. The estimates are presented in the right column of Table 6. Both coefficients of MALE and HINCOME are negative, but they are not significant at all. Thus, for our subjects, the discount rates are independent of these attributes. On the other hand, the three anomalies are clearly confirmed with this specification. The result that $P A Y$ has no effect also remains unchanged.

\subsection{Robustness check on our time discounting data}

We next examine the reliability of the discount rates elicited in the experiment. In the questionnaire that we asked subjects to complete at the end of the experiment, questions similar to those in the experiment were included. In particular, we asked the following five questions, each of which has a counterpart "round" in the experiment.

QTD5, which corresponds to round 5: $s=2$ days, $h=7$ days, but $X=800$ yuan instead of 2800 yuan. 
QTD6, which corresponds to round 6: $s=90$ days, $h=7$ days, but $X=800$ yuan instead of 2800 yuan.

QTD3\&8, which corresponds to rounds 3 and 8: $s=1$ month, $h=12$ months, but $X=$ 800 yuan instead of 240 and 2800 yuan.

QTD11, which corresponds to round 11: $s=1$ month, $h=12$ months, and $X=$ 800000 yuan.

We examine whether or not these four sets of data elicited by the questionnaire are consistent with the discount rates obtained from the experiment. The mean and standard deviation of the logarithm of the discount rates elicited by the questionnaire $(Q T D)$ and the experiment (ETD) are presented together with the experimental conditions, in the upper and middle panels of Table 7, respectively. Comparing QTD5 with the corresponding ETD5, and QTD6 with ETD6, the former is higher than the latter, whereas the discount rate of QTD3\&8 is lower than ETD3 and higher than ETD8. Thus, the discount rates with a larger reward amount are consistently lower. As we noted above, the corresponding pairs are different only in the reward amount, and the results correspond to the magnitude effect that we found in section 3.2. Thus, although the test of the same mean rejects the null of the same mean, the mean may be the same after we adjust the magnitude effect.

To examine whether this is the case, we estimate the following equation (2), which differs from equation (1) only in that the logarithm of the reward amount is used here, instead of the amount dummies.

$$
\begin{aligned}
\ln T D_{i j}= & \alpha_{0}+\alpha_{1} \mathrm{HOR}_{j}+\alpha_{2} \mathrm{HOR}_{3} 0_{j}+\alpha_{3} \mathrm{HOR}_{j}+\alpha_{4} I N T_{j}+\alpha_{5} A M T_{j} \\
& +\alpha_{7} P A Y_{j}+\varepsilon_{i, j}
\end{aligned}
$$

where $A M T$ is equal to either $\log 240, \log 2800$, or $\log 800000$. Then, the predicted value 
of the discount rates from the experiment, $A D J T D$, is calculated by the following equation (3). For example, in the case of round 5, if the amount were set at 800 yuan instead of the actual 2800 yuan and the payment was not promised, the discount rate would be

$$
A D J T D_{i, 5} \equiv \ln T D_{i, 5}+\hat{\alpha}_{5}(\log 800-\log 2800)-\hat{\alpha}_{7}
$$

The mean and standard deviation of the values thus calculated are presented in Panel $\mathrm{C}$ of Table 7. The ADJTD values move closer to the discount rates from the questionnaire, as expected. Although the statistical test (Panel D of Table 7) rejects the null of the same mean between QTD5 and ADJTD5, and between QTD6 and ADJTD6, the null that QTD3\&8 and ADJTD3 are the same is not rejected at the $30 \%$ level, and the null associated with QTD3\&8 and ADJTD8 is not rejected at the 5\% level. Thus, although the discount rates elicited by the questionnaire and the experiment are not exactly the same, in some cases they are close enough.

The consistency between the discount rates elicited by the experiment and by the questionnaire is confirmed by another analysis. Table 8 presents the correlation coefficient between these discount rates and reveals that the correlation between the pairs of experimental conditions that are similar is relatively higher (highlighted in yellow) than that of the other pairs, implying that the subjects respond consistently to similar questions.

These results indicate that the time discount rates elicited from the experiment are generally consistent with those elicited from the questionnaire, and that our experimental data are reliable.

\section{Time discounting and procrastinating behavior}




\subsection{Analytical framework}

People are sometimes unable to behave as they had originally planned. Instead, they tend to postpone discomfort and enjoy pleasure earlier. In this section, we investigate whether the subjects' time preference, as revealed in the experiment, can explain their procrastinating behavior.

As an example of an unpleasant task in which people tend to procrastinate, we adopt homework assignments in childhood. In the questionnaire distributed at the end of the experiment, we asked when they did their assignments:

"Thinking about when you were a child and you were given an assignment in school, when did you usually do the assignment?" (Circle ONE number)

1. Got it done right away

2. Tended to get it done early, before the due date

3. Worked on it daily up until the due date

4. Tended to get it done toward the end

5. Got it done at the last minute

From their answers, we define $H W K_{i}$ as the number that subject $i$ chose. A larger value of $H W K_{i}$ means a stronger propensity to procrastinate.

Our hypotheses are as follows. First, higher time discounting will lead to procrastinating behavior. Second, the delay effect may reinforce procrastinating behavior. A stronger delay effect means higher time discounting in the near future and/or lower discounting in the remote future. Therefore, controlling the average discount rate in the regression, a stronger delay effect implies higher time discounting in the near future, so that it seems to bring about procrastinating behavior. However, the case may not be so simple. The delay effect causes a preference reversal, so that people 
subject to the delay effect often procrastinate, and then regret that they had procrastinated. Thus, after repeatedly suffering bitter regret, they may devise a way to prevent procrastination, in order to avoid having to regret a miserable outcome. If this is so, the stronger delay effect no longer results in greater procrastination. ${ }^{7}$

Following O’Donoghue and Rabin (1999), let us categorize individuals who show the delay effect into two types: naïfs and sophisiticates. Naïfs mistakenly believe that they will behave as they originally planned, and so they delay imminent costs and enjoy immediate rewards. On the other hand, sophisticates know that they will delay an unpleasant task until the date of the execution that was originally planned comes and that such procrastination may lead to a disaster in the end; therefore, they may undertake an unpleasant task now (Proposition 2 of O'Donoghue and Rabin, 1999).

Given these arguments, we speculate that (i) if the subjects are naïfs, the delay effect will enhance their procrastination; (ii) however, if they are sophisticates, the delay effect may not promote procrastination clearly; it may even suppress procrastination.

Thus, our regression equation is

$$
H W K_{i}=\beta+\gamma T D_{i}+\delta D L Y_{i}+u_{i}, \quad i=1, \ldots, 29 .
$$

We expect $\gamma>0$. However the sign of $\delta$ is not known a priori. It depends on whether the subjects are naïfs or sophisticates.

\subsection{Definition of variables used in the regression}

\footnotetext{
7 Here we assume that slight procrastination does not bring about sufficient regret to stop further procrastination, even by sophisticates.
} 
By construction, the relation should be estimated with individual data, so that we need to use the time discount rate and the degree of the delay effect for each person. Thus, the time discount rate of subject $i, A V T D_{i}$, is defined as the average of the logarithms of the discount rates reported by subject $i$ in round $j, L N T D_{i j}$ : that is,

$$
A V T D_{i}=\sum_{j=1}^{12} L N T D_{i j} / 12
$$

However, the simple average of the time discount rates elicited in different conditions may suffer from a problem. For example, we saw in Table 1 that the time discount rate in the case of large reward amount of 800000 yuan is much smaller than in the case of smaller reward amounts. Therefore, the difference in the discount rate between subjects in the case of the large reward amount is substantially smaller than in the case of the small reward amount. Thus, a simple average would underestimate the contribution of the discount rates in the case of the large reward amount. To be free from this bias, we first standardize logarithmic discount rates in each round to obtain $\operatorname{STDTD}_{i j}=\left(\frac{L N T D_{i j}-\mu_{j}}{\sigma_{j}}\right)$, where $\mu_{j}$ and $\sigma_{j}$ are the mean and standard deviation of logarithmic time discount rates in round $j$ over the subjects. Then, the standardized time discount rate for each subject is defined as

$$
\operatorname{AVSTDTD}_{i}=\sum_{j=1}^{12} \operatorname{STDTD}_{i j} / 12
$$

On the other hand, we define the degree of the delay effect of subject $i, D L Y$, as the time discount rate in the case of 2-days delay subtracted from that of 90-days delay, that is, 


$$
D L Y_{i}=L N T D_{i 5}-L N T D_{i 6}
$$

because, as we confirmed in section 3.2, the delay effect exists only between these delays. Nonetheless, to check the robustness of our conclusions, we report the results using STDDLY, which is defined as the average of the differences in standardized time discount rates, that is,

$$
\begin{aligned}
\operatorname{STDDLY}_{i}= & {\left[\left(\operatorname{STDTD}_{i 2}-\operatorname{STDTD}_{i 4}\right)+\left(\operatorname{STDTD}_{i 5}-\operatorname{STDTD}_{i 6}\right)\right.} \\
& \left.+\left(\operatorname{STDTD}_{i 7}-\operatorname{STDTD}_{i 9}\right)+\left(\operatorname{STDTD}_{i 10}-\operatorname{STDTD}_{i 12}\right)\right] / 4 .
\end{aligned}
$$

\subsection{Estimation results on procrastination}

Equation (4) is estimated by ordered probit because the dependent variable takes on integers from one to five, and a larger value represents stronger procrastination. The results using $D L Y_{i}$ as the degree of the delay effect are presented in the upper panel of Table 9. In the left columns, $H W K_{i}$ is regressed only over the time discount rate, which reveals that the higher discount rates enhance the procrastination. ${ }^{8}$ Whether or not the discount rates are standardized does not change the results. The middle column of the table shows the estimates in the case in which $H W K_{i}$ is regressed only over the degree of the delay effect, $D L Y_{i}$. Although $D L Y_{i}$ has a negative coefficient, it is not significant at all. In the columns on the right, $H W K_{i}$ is regressed over both the time discount rate and the degree of the delay effect, confirming the above results: although the variables representing the time discount rate are significant at the $1 \%$ or $5 \%$ level, the coefficient of the degree of the delay effect $D L Y_{i}$ is negative, but not significant at all.

\footnotetext{
${ }^{8}$ Note that the estimated coefficients do not represent the marginal effect on the value of the HWK. They represent the effect on the continuous latent variable corresponding to the HWK.
} 
In the rightmost column of Table 9, we show the results using STDDLY instead of $D L Y$. The time discount rates have similar coefficients, but the coefficient of STDDLY is significantly negative. In either case, the results that the coefficients of the degree of the delay effect are not positive suggest that our subjects may be sophisticates.

One might suspect, however, that our results merely failed to reflect the reality, and are thus not reliable. To address this concern, we conduct an additional analysis. Following the model of naïfs and sophisticates in O'Donoghue and Rabin (1999), and assuming that slight procrastination does not bring about regret strong enough to prevent further procrastination, even by sophisticates, we examine a hypothesis that consists of the following elements (see Table 10):

1) Among the naïfs, a higher delay effect brings about stronger procrastination (right column of Table 10).

2) Among the sophisticates with a high delay effect, the effect of the delay effect is not known a priori: the magnitude of the delay effect may not affect procrastination at all or it may even affect it negatively (left-upper cell of Table 10).

3) Among the sophisticates with a low delay effect, a higher delay effect may or may not bring about stronger procrastination (left-bottom cell of Table $10)$.

However, the hypothesis is not refutable, because we have not defined who are naïfs and who are sophisticates independently of the degree of the delay effect. It is natural to assume that sophisticates are more intellectual and introspective persons. Thus, in this study, we assume that all of our subjects are sophisticates, since the students of Fudan 
University are undoubtedly the top elite in China.

To substantiate our interpretation, we define a variable representing the degree of the delay effect of the higher group, $H D E_{\mathrm{i}}$, and a variable representing that of the lower group, $L D E_{\mathrm{i}}$, as follows:

$$
\begin{aligned}
H D E_{i} & \equiv\left\{\begin{array}{ccl}
D L Y_{i} & \text { if } & D L Y_{i} \geq \overline{D L Y} \\
0 & \text { otherwise }
\end{array}\right. \\
L D E_{i} & \equiv\left\{\begin{array}{cll}
D L Y_{i} & \text { if } & D L Y_{i}<\overline{D L Y} \\
0 & \text { otherwise }
\end{array},\right.
\end{aligned}
$$

where $\overline{D L Y}$ stands for a critical value dividing the subjects into two groups that will be specified below. The regression equation now becomes

$$
H W K_{i}=\beta+\gamma T D_{i}+\delta_{H} H D E_{i}+\delta_{L} L D E_{i}+v_{i}, \quad i=1, \ldots, 29 .
$$

Our hypothesis is $\delta_{H} \leq \delta_{L} \geq 0$. On the contrary, if our subjects are all naïfs, then $\delta_{H}=\delta_{L} \geq 0$ will be obtained (see the middle panel of Table 10).

To determine the critical value $\overline{D L Y}$, let us take a look at the histogram of $D L Y$ in Figure 1. It reveals that there are two spikes, at 0 and 0.74 . We do not have a clear idea of how large the critical value that separates naïfs from sophisticates is. Thus, we arbitrarily adopt $0.7,0.9$, and 1.0 , each of which gives divisions of the samples into (high group, low group $)=(12,17),(6,23),(4,25)$, respectively. The estimation results of equation (11) are presented in the upper panel of Table 10. When the critical value is set at 0.7 (the left column), the coefficient of the degree of the delay effect of the high group, $H D E$, is negative, and that of the low group, $L D E$, is positive, as expected. However, both of them are not significant. When we separate the groups at the value of 
0.9 , the coefficient of the high group becomes smaller and significant at the $10 \%$ level (see the middle column). When the critical value is set at 1.0 , the coefficient of the high group again becomes smaller, with the p-value of $5.7 \%$ (the right column). The coefficient of the low group becomes larger, and its p-value also becomes smaller as the critical value is raised, although it does not become significant. Meanwhile, the time discount rate, AVTD, is significant at the $5 \%$ level in all cases. These results provide some evidence for the naïfs-sophisticates interpretation of our results. In sum, we obtain $\delta_{H}<0$ and $\delta_{L}=0$.

In the lower panel of Table 11, we present the results using the average of the degree of the delay effect, $S T D D L Y$, instead of the degree of the delay effect within the short delays, DLY. The histogram of STDDLY shown in Figure 2 reveals that 15 out of 29 subjects do not report the delay effect, if this variable is taken as the indicator of the delay effect. Thus, we first stratify all the subjects who report negative STDDLY as the low-delay-effect group and all others as the high-delay-effect group, and define the degree of the delay effect of the high group, HSTDDLY, and that of the low group, LSTDDLY. The estimates in this case are presented in the left column, and they reveal that the high group (HSTDDLY) has a significant negative coefficient and the low group ( $L S T D D L Y)$ has a positive coefficient, which is almost significant at the $10 \%$ level. Thus, we again obtain $\delta_{H}<0$ and $\delta_{L}=0$. When we raise the critical value to 0.1 , the difference between these coefficients becomes smaller, which suggests that the critical value should be smaller. ${ }^{9}$ All the results in Table 11 are consistent with our speculation, i.e., the naïfs-sophisticates model.

\footnotetext{
${ }^{9}$ However, we should be careful about the interpretation of the magnitude, because the value is not the marginal effect.
} 
However, we should note that our analysis does not offer abundant evidence for the naïfs-sophisticates model. This study has, at best, found that the model seems to hold for a specific sample of the students at Fudan University, while many studies, including Hiruma and Ikeda (2007) and Tsutsui et al. (2007) have found a positive correlation between the degree of the delay effect and the loan amount. The naïfs-sophisticates model interprets this as the former results being obtained for sophisticates and the latter results for naïfs, but this interpretation may seem arbitrary. To confirm that the model describes the reality, it is necessary to find a negative correlation between the degree of the delay effect and procrastination in more cases. In addition, although our presumption that our subjects are all sophisticates may be reasonable because they are all students of the top university, it is still necessary to examine our hypothesis presented in Table 10, using broader data consisting of both naïfs and sophisticates.

\section{Conclusion}

This study investigated the time discounting of human beings by an economic experiment. Economic experiments are often termed as unreliable because the subjects recruited do not have sufficient incentives to report their true preference. Our experiment is immune to such criticism because the highest reward shown to the subjects is close to their monthly household income.

This study achieved two goals. First, the delay effect people discount the near future more than the remote future is substantiated by explicitly separating the delay 
from the interval. ${ }^{10}$ Most previous studies have not separated these two experimental conditions, while Read (2001) identified these two conditions and reported that the delay effect was not found. Setting shorter delays than Read, we substantiated the delay effect for a delay of less than 90 days. This finding is important because the delay effect induces people to make time-inconsistent plans and then to regret their decision.

Another contribution of this study is its explanation of the subjects' procrastinating behavior by their time discount rates and the degree of the delay effect. Higher time discounting always promote procrastination, but the delay effect involves two competing consequences for procrastination: it has a positive effects when it is not strong, but it reduces procrastination when it is strong. Our results are consistent with the naïfs-sophisticates model developed by O'Donoghue and Rabin (1999).

\footnotetext{
${ }^{10}$ At the same time, we confirmed the interval effect, in which subjects discount the future more in shorter intervals than in longer intervals, and the magnitude effect, in which they discount the future more for smaller amounts of money.
} 


\section{References}

Ainslie, George (1975), Specious Reward: A Behavioral Theory of Impulsiveness and Impulse Control, Psychological Bulletin, 82 (4), 463-96.

Benzion, Uri, Amnon Rapaport, and Joseph Yagil (1989), Discount Rates Inferred from Decisions: An Experimental Study, Management Science, 35, 270-84.

Frederick, Shane, George Loewenstein and Ted O'Donoghue (2002), Time Discounting and Time Preference: A Critical Review, Journal of Economic Literature, 40, $351-401$.

Hiruma, Fumihiko and Shinsuke Ikeda (2007) “A Study on Time Discounting Based on Experiments," Review of Monetary and Financial Studies, 25, 12-25. (in Japanese)

Kirby, Kris N. (1997), Bidding on the Future: Evidence Against Normative Discounting of Delayed Rewards, Journal of Experimental Psychology: General, 126, 54-70.

Kirby, Kris N. and Nino N. Marakovic (1995), Modeling Myopic Decisions: Evidence for Hyperbolic Delay-Discounting with Subjects and Amounts, Organizational Behavior and Human Decision Processes, 64, 22-30.

Laibson, David (1997), Golden Eggs and Hyperbolic Discounting, Quarterly Journal of Economics, 112, 443-77.

O’Donoghue, Ted and Matthew Rabin (1999), Doing It Now or Later, American Economic Review, 89 (1), 103-24.

Read, Daniel (2001), Is Time Discounting Hyperbolic or Subadditive?, Journal of Risk and Uncertainty, 23, 5-32.

Read, Daniel, and Peter H. M. P. Roelofsma (2003), Subadditive versus Hyperbolic Discounting: A Comparison of Choice and Matching, Organizational Behavior and Human Decision Process, 91, 140-153.

Read, Daniel, Shane Frederick, Burcu Orsel, and Juwaria Rahman (2005), Four Score and Seven Years from Now: The Date/Delay Effect in Temporal Discounting, Management Science, 51, 1326-35.

Sasaki, Shinichiro, Shiyu Xie, Fumio Ohtake, Jie Qin, and Yoshiro Tsustui (2008), Experiments on Risk Attitude: the Case of Chinese Students, China Economic Review, 19 (2), 245-59.

Thaler, Richard H. (1981), Some Empirical Evidence on Dynamic Inconsistency, 
Economic Letters, 8, 201-7.

Tsutsui, Yoshiro, Fumihiko Hiruma, Fumio Ohtake, and Sinsuke Ikeda (2007) "An Analysis of the Impact of Interest Rate Ceilings to the Consumer Credit: Behavioral Economic Approach," Gendai Finance, 22, 25-73. (in Japanese) 
Table 1. Experimental conditions and mean discount rates

\begin{tabular}{ccccccc}
\hline Round & 1 & 2 & 3 & 4 & 5 & 6 \\
\hline Delay $(s)$ & 1 month & 1 month & 1 month & 10 months & 2 days & 90 days \\
Interval $(h)$ & 3 months & 3 months & 1 year & 3 months & 7 days & 7 days \\
Rewards $(X)$ & 2800 & 240 & 240 & 240 & 2800 & 2800 \\
Payment $(P)$ & No & Yes & Yes & Yes & Yes & Yes \\
Mean discount rate & 9.586 & 40.034 & 23.793 & 43.828 & 30.638 & 22.121 \\
Standard deviation & 11.228 & 40.498 & 20.304 & 46.475 & 49.328 & 37.841 \\
\hline \multicolumn{7}{c}{} \\
\hline Round & 7 & 8 & 9 & 10 & 11 & 12 \\
\hline Delay $(s)$ & 1 month & 1 month & 10 months & 1 month & 1 month & 10 months \\
Interval $(h)$ & 3 months & 1 year & 3 months & 3 months & 1 year & 3 months \\
Rewards $(X)$ & 2800 & 2800 & 2800 & 800000 & 800000 & 800000 \\
Pay $(P)$ & Yes & Yes & Yes & No & No & No \\
Mean discount rate & 8.276 & 5.819 & 9.034 & 1.103 & 1.491 & 0.959 \\
Standard deviation & 6.866 & 5.105 & 10.987 & 2.033 & 3.525 & 1.612 \\
\hline
\end{tabular}


Table 2. Discount rates' and delays

\begin{tabular}{|c|c|c|c|c|c|c|c|c|}
\hline Round & 2 & 4 & 5 & 6 & 7 & 9 & 10 & 12 \\
\hline Delay $(s)$ & 1 month & 300 & 2 days & 90 days & 1 month & 300 & 1 month & 10 months \\
\hline Interval $(h)$ & 3 months & 3 months & 7 days & 7 days & 3 months & 3 months & 3 months & 3 months \\
\hline Rewards $(X)$ & 240 & 240 & 2800 & 2800 & 2800 & 2800 & 800000 & 800000 \\
\hline Payment $(P)$ & Yes & Yes & Yes & Yes & Yes & Yes & No & No \\
\hline Observations & 29 & 29 & 29 & 29 & 29 & 29 & 29 & 29 \\
\hline $\begin{array}{l}\text { Mean discount } \\
\text { rate }\end{array}$ & 40.034 & 43.828 & 30.638 & 22.121 & 8.276 & 9.034 & 1.103 & 0.959 \\
\hline $95 \%$ upper bound & 24.630 & 26.149 & 11.875 & 7.727 & 5.664 & 4.855 & 0.329 & 0.346 \\
\hline $95 \%$ lower bound & 55.439 & 61.506 & 49.401 & 36.514 & 10.887 & 13.214 & 1.876 & 1.573 \\
\hline P-value & \multicolumn{2}{|c|}{0.617} & \multicolumn{2}{|c|}{0.317} & \multicolumn{2}{|c|}{0.455} & \multicolumn{2}{|c|}{0.638} \\
\hline
\end{tabular}


Table 3. Discount rates and intervals

\begin{tabular}{|c|c|c|c|c|c|c|}
\hline Round & 2 & 3 & 7 & 8 & 10 & 11 \\
\hline Delay (s) & 1 month & 1 month & 1 month & 1 month & 1 month & 1 month \\
\hline Interval $(h)$ & 3 months & 1 year & 3 months & 1 year & 3 months & 1 year \\
\hline Rewards $(X)$ & 240 & 240 & 2800 & 2800 & 800000 & 800000 \\
\hline Payment $(P)$ & Yes & Yes & Yes & Yes & No & No \\
\hline Observations & 29 & 29 & 29 & 29 & 29 & 29 \\
\hline $\begin{array}{l}\text { Mean discount } \\
\text { rate }\end{array}$ & 40.034 & 23.793 & 8.276 & 5.819 & 1.103 & 1.491 \\
\hline $95 \%$ upper bound & 24.630 & 16.070 & 5.664 & 3.877 & 0.329 & 0.151 \\
\hline $95 \%$ lower bound & 55.439 & 31.516 & 10.887 & 7.761 & 1.876 & 2.832 \\
\hline P-value & \multicolumn{2}{|c|}{0.003} & \multicolumn{2}{|c|}{0.003} & \multicolumn{2}{|c|}{0.538} \\
\hline
\end{tabular}


Table 4. Discount rates and reward amounts

\begin{tabular}{|c|c|c|c|c|c|c|}
\hline Round & 2 & 7 & 3 & 8 & 4 & 9 \\
\hline Delay $(s)$ & 30 & 30 & 30 & 30 & 300 & 300 \\
\hline Interval $(h)$ & 90 & 90 & 365 & 365 & 90 & 90 \\
\hline Rewards $(X)$ & 240 & 2800 & 240 & 2800 & 240 & 2800 \\
\hline Payment $(P)$ & Yes & Yes & Yes & Yes & Yes & Yes \\
\hline Observations & 29 & 29 & 29 & 29 & 29 & 29 \\
\hline $\begin{array}{l}\text { Mean discount } \\
\text { rate }\end{array}$ & 40.034 & 8.276 & 23.793 & 5.819 & 43.828 & 9.034 \\
\hline $95 \%$ upper bound & 24.63 & 5.664 & 16.07 & 3.877 & 26.149 & 4.855 \\
\hline $95 \%$ lower bound & 55.439 & 10.887 & 31.516 & 7.761 & 61.506 & 13.214 \\
\hline P-value & \multicolumn{2}{|c|}{0.000} & \multicolumn{2}{|c|}{0.000} & \multicolumn{2}{|c|}{0.000} \\
\hline Round & 7 & 10 & 8 & 11 & 9 & 12 \\
\hline Delay $(s)$ & 30 & 30 & 30 & 30 & 300 & 300 \\
\hline Interval $(h)$ & 90 & 90 & 365 & 365 & 90 & 90 \\
\hline Rewards $(X)$ & 2800 & 800000 & 2800 & 800000 & 2800 & 800000 \\
\hline Payment $(P)$ & Yes & No & Yes & No & Yes & No \\
\hline Observations & 29 & 29 & 29 & 29 & 29 & 29 \\
\hline $\begin{array}{l}\text { Mean discount } \\
\text { rate }\end{array}$ & 8.276 & 1.103 & 5.819 & 1.491 & 9.034 & 0.959 \\
\hline $95 \%$ upper bound & 5.664 & 0.329 & 3.877 & 0.151 & 4.855 & 0.346 \\
\hline $95 \%$ lower bound & 10.887 & 1.876 & 7.761 & 2.832 & 13.214 & 1.573 \\
\hline P-value & \multicolumn{2}{|c|}{0.000} & \multicolumn{2}{|c|}{0.001} & \multicolumn{2}{|c|}{0.001} \\
\hline Round & 2 & 10 & 3 & 11 & 4 & 12 \\
\hline Delay $(s)$ & 30 & 30 & 30 & 30 & 300 & 300 \\
\hline Interval $(h)$ & 90 & 90 & 365 & 365 & 90 & 90 \\
\hline Rewards $(X)$ & 240 & 800000 & 240 & 800000 & 240 & 800000 \\
\hline Payment $(P)$ & Yes & No & Yes & No & Yes & No \\
\hline Observations & 29 & 29 & 29 & 29 & 29 & 29 \\
\hline $\begin{array}{l}\text { Mean discount } \\
\text { rate }\end{array}$ & 40.034 & 1.103 & 23.793 & 1.491 & 43.828 & 0.959 \\
\hline $95 \%$ upper bound & 24.63 & 0.329 & 16.07 & 0.151 & 26.149 & 0.346 \\
\hline $95 \%$ lower bound & 55.439 & 1.876 & 31.516 & 2.832 & 61.506 & 1.573 \\
\hline P-value & \multicolumn{2}{|c|}{0.000} & \multicolumn{2}{|c|}{0.000} & \multicolumn{2}{|c|}{0.000} \\
\hline
\end{tabular}


Table 5. Discount rates and the payment of the reward

\begin{tabular}{ccc}
\hline Round & 1 & 7 \\
\hline Delay $(s)$ & 30 & 30 \\
Interval $(h)$ & 90 & 90 \\
Rewards $(X)$ & 2800 & 2800 \\
Payment $(P)$ & No & Yes \\
Observations & 29 & 29 \\
Mean discount rate & 9.586 & 8.276 \\
95\% upper bound & 5.315 & 5.664 \\
95\% lower bound & 13.857 & 10.887 \\
P-value & \multicolumn{2}{c}{0.461} \\
\hline
\end{tabular}


Table 6. Result of the regression analysis on three anomalies

\begin{tabular}{|c|c|c|c|c|c|c|}
\hline & $\begin{array}{l}\text { Estimated } \\
\text { coefficient }\end{array}$ & P-value & $\begin{array}{l}\text { Estimated } \\
\text { coefficient }\end{array}$ & P-value & $\begin{array}{l}\text { Estimated } \\
\text { coefficient }\end{array}$ & P-value \\
\hline Constant & -1.074 & 0.000 & -1.070 & 0.000 & 6.067 & 0.000 \\
\hline HOR2 & 0.765 & 0.004 & 0.733 & 0.010 & 0.572 & 0.053 \\
\hline HOR30 & 0.077 & 0.651 & 0.198 & 0.276 & 0.072 & 0.712 \\
\hline HOR90 & 0.336 & 0.203 & 0.297 & 0.295 & 0.249 & 0.400 \\
\hline АMT240 & 4.413 & 0.000 & 4.518 & 0.000 & & \\
\hline AMT2800 & 2.941 & 0.000 & 3.113 & 0.000 & & \\
\hline $\log (A M T)$ & & & & & -0.526 & 0.000 \\
\hline INT & -0.001 & 0.018 & -0.002 & 0.012 & -0.001 & 0.044 \\
\hline$P A Y$ & 0.001 & 0.997 & -0.029 & 0.918 & 0.064 & 0.829 \\
\hline$M A L E$ & & & -0.016 & 0.971 & & \\
\hline HINCOME & & & -0.148 & 0.720 & & \\
\hline $\begin{array}{l}\text { Number of } \\
\text { observations }\end{array}$ & \multicolumn{2}{|c|}{348} & \multicolumn{2}{|c|}{276} & \multicolumn{2}{|c|}{348} \\
\hline $\begin{array}{c}\text { Number of } \\
\text { subjects }\end{array}$ & \multicolumn{2}{|c|}{29} & \multicolumn{2}{|c|}{23} & \multicolumn{2}{|c|}{29} \\
\hline $\mathrm{R}^{2}$ & \multicolumn{2}{|c|}{0.6284} & \multicolumn{2}{|c|}{0.6525} & \multicolumn{2}{|c|}{0.616} \\
\hline
\end{tabular}

Note: regression equation is

$\ln T D_{i j}=\alpha_{0}+\alpha_{1}$HOR $_{j}+\alpha_{2}{\text { HOR } 30_{j}}_{j}+\alpha_{3}$ HOR $0_{j}+\alpha_{4} I N T_{j}+\alpha_{5} A M T 240_{j}+\alpha_{6}$ AMT2800 $_{j}+\alpha_{7} P A Y_{j}+\varepsilon_{i, j}$ 
Table 7. Comparisons of discount rates in the questionnaire and the experiment

Panel A: Questionnaire

\begin{tabular}{ccccc}
\hline & QTD5 & QTD6 & QTD3\&8 & QTD11 \\
\hline Delay $(s)$ & 2 days & 90 days & 1 month & 1 month \\
Interval $(h)$ & 7 days & 7 days & 1 year & 1 year \\
Rewards $(X)$ & 800 & 800 & 800 & 800000 \\
Payment $(P)$ & No & No & No & No \\
QTD: Mean & 3.809 & 3.677 & 2.271 & 0.004 \\
Standard Deviation & 1.294 & 1.278 & 0.765 & 1.145 \\
Standard error & 0.249 & 0.242 & 0.145 & 0.213 \\
95\% upper bound & 3.297 & 3.181 & 1.974 & -0.432 \\
95\% lower bound & 4.321 & 4.173 & 2.568 & 0.439 \\
\hline
\end{tabular}

Panel B: Experiment

\begin{tabular}{cccccc}
\hline & ETD5 & ETD6 & ETD3 & ETD8 & ETD11 \\
\hline Delay $(s)$ & 2 days & 90 days & 1 month & 1 month & 1 month \\
Interval $(h)$ & 7 days & 7 days & 1 year & 1 year & 1 year \\
Rewards $(X)$ & 2800 & 2800 & 240 & 2800 & 800000 \\
Payment $(P)$ & Yes & Yes & Yes & Yes & No \\
ETD: Mean & 2.622 & 2.194 & 2.816 & 1.392 & -1.425 \\
Standard Deviation & 1.386 & 1.468 & 0.892 & 0.962 & 1.991 \\
Standard error & 0.257 & 0.273 & 0.166 & 0.179 & 0.370 \\
95\% upper bound & 2.095 & 1.635 & 2.477 & 1.026 & -2.182 \\
95\% lower bound & 3.149 & 2.752 & 3.156 & 1.758 & -0.667 \\
\hline
\end{tabular}

Panel C: Adjusted discount rates

\begin{tabular}{ccccc}
\hline & ADJTD5 & ADJTD6 & ADJTD3 & ADJTD8 \\
\hline Delay $(s)$ & 2 days & 90 days & 1 month & 1 month \\
Interval $(h)$ & 7 days & 7 days & 1 year & 1 year \\
Rewards $(X)$ & 2800 & 2800 & 240 & 2800 \\
Payment $(P)$ & Yes & Yes & Yes & Yes \\
ADJTD: Mean & 2.789 & 2.789 & 2.119 & 1.988 \\
Standard deviation & 1.386 & 1.468 & 0.892 & 0.962 \\
Standard error & 0.257 & 0.273 & 0.166 & 0.179 \\
95\% upper bound & 2.262 & 2.231 & 1.780 & 1.622 \\
95\% lower bound & 3.316 & 3.347 & 2.458 & 2.354 \\
\hline
\end{tabular}

Notes: QTD and ETD are the logarithm of the discount rates elicited by the questionnaire and the experiment, respectively. Adjusted discount rates are calculated from the regression shown in the rightmost column of Table 6 . 
Panel D: Mean comparison test of the discount rate in the questionnaire and the experiment

\begin{tabular}{|c|c|c|c|c|c|c|}
\hline Compared group & QTD5 & ADJTD5 & QTD6 & ADJTD6 & QTD3\&8 & ADJTD3 \\
\hline Number of Observations & 29 & 27 & 29 & 28 & 29 & 28 \\
\hline P-value & \multicolumn{2}{|c|}{0.000} & \multicolumn{2}{|c|}{0.001} & \multicolumn{2}{|c|}{0.303} \\
\hline Comparison & QTD3\&8 & ADJTD8 & QTD11 & ETD11 & & \\
\hline Number of Observations & 29 & 29 & 29 & 29 & & \\
\hline P-value & & & & & & \\
\hline
\end{tabular}


Table 8. Correlation between QTD and ETD

\begin{tabular}{|c|c|c|c|c|c|c|c|c|c|}
\hline & QTD5 & QTD6 & QTD3\&8 & QTD11 & ETD5 & ETD6 & ETD3 & ETD8 & ETD11 \\
\hline QTD5 & 1.000 & & & & & & & & \\
\hline QTD6 & $\begin{array}{c}0.901 \\
(0.000)\end{array}$ & 1.000 & & & & & & & \\
\hline QTD3\&8 & $\begin{array}{c}0.545 \\
(0.003)\end{array}$ & $\begin{array}{c}0.417 \\
(0.027)\end{array}$ & 1.000 & & & & & & \\
\hline QTD11 & $\begin{array}{l}-0.067 \\
(0.738)\end{array}$ & $\begin{array}{l}-0.149 \\
(0.450)\end{array}$ & $\begin{array}{c}0.174 \\
(0.375)\end{array}$ & 1.000 & & & & & \\
\hline ETD5 & $\begin{array}{c}0.700 \\
(0.000)\end{array}$ & $\begin{array}{c}0.580 \\
(0.001)\end{array}$ & $\begin{array}{c}0.345 \\
(0.072)\end{array}$ & $\begin{array}{c}0.102 \\
(0.598)\end{array}$ & 1.000 & & & & \\
\hline ETD6 & $\begin{array}{c}0.733 \\
(0.000)\end{array}$ & $\begin{array}{c}0.751 \\
(0.000)\end{array}$ & $\begin{array}{c}0.414 \\
(0.029)\end{array}$ & $\begin{array}{c}0.014 \\
(0.941)\end{array}$ & $\begin{array}{c}0.697 \\
(0.000)\end{array}$ & 1.000 & & & \\
\hline ETD3 & $\begin{array}{c}0.340 \\
(0.082)\end{array}$ & $\begin{array}{c}0.234 \\
(0.232)\end{array}$ & $\begin{array}{c}0.354 \\
(0.065)\end{array}$ & $\begin{array}{c}0.058 \\
(0.765)\end{array}$ & $\begin{array}{c}0.297 \\
(0.117)\end{array}$ & $\begin{array}{c}0.375 \\
(0.045)\end{array}$ & 1.000 & & \\
\hline ETD8 & $\begin{array}{c}0.256 \\
(0.198)\end{array}$ & $\begin{array}{c}0.088 \\
(0.655)\end{array}$ & $\begin{array}{c}0.557 \\
(0.002)\end{array}$ & $\begin{array}{c}0.282 \\
(0.138)\end{array}$ & $\begin{array}{c}0.333 \\
(0.077)\end{array}$ & $\begin{array}{c}0.297 \\
(0.127)\end{array}$ & $\begin{array}{c}0.536 \\
(0.003)\end{array}$ & 1.000 & \\
\hline ETD11 & $\begin{array}{l}-0.144 \\
(0.475)\end{array}$ & $\begin{array}{l}-0.232 \\
(0.235)\end{array}$ & $\begin{array}{c}0.056 \\
(0.775)\end{array}$ & $\begin{array}{c}0.838 \\
(0.000)\end{array}$ & $\begin{array}{l}-0.062 \\
(0.751)\end{array}$ & $\begin{array}{l}-0.171 \\
(0.374)\end{array}$ & $\begin{array}{c}0.067 \\
(0.730)\end{array}$ & $\begin{array}{c}0.296 \\
(0.120)\end{array}$ & 1.000 \\
\hline
\end{tabular}

Notes: QTD and ETD are the logarithm of the discount rates elicited by the questionnaire and the experiment, respectively. The cells highlighted in yellow indicate the correlation between the pairs of experimental conditions that are similar. P-values are in parentheses. 
Table 9. Time discounting and procrastination

\begin{tabular}{|c|c|c|c|c|c|c|c|c|c|c|c|c|}
\hline & $\begin{array}{l}\text { Estimated } \\
\text { coefficient }\end{array}$ & P-value & $\begin{array}{l}\text { Estimated } \\
\text { coefficient }\end{array}$ & $\mathrm{P}$-value & $\begin{array}{l}\text { Estimated } \\
\text { coefficient }\end{array}$ & P-value & $\begin{array}{l}\text { Estimated } \\
\text { coefficient }\end{array}$ & $\mathrm{P}$-value & $\begin{array}{l}\text { Estimated } \\
\text { coefficient }\end{array}$ & P-value & $\begin{array}{l}\text { Estimated } \\
\text { coefficient }\end{array}$ & $\mathrm{P}$-value \\
\hline Constant & 0.331 & 0.487 & 1.394 & 0.000 & 1.278 & 0.000 & 0.357 & 0.459 & 1.413 & 0.000 & 1.699 & 0.000 \\
\hline$A V T D$ & 0.794 & 0.009 & & & & & 0.803 & 0.008 & & & & \\
\hline AVSTDTD & & & 0.774 & 0.030 & & & & & 0.775 & 0.030 & 1.535 & 0.001 \\
\hline$D L Y$ & & & & & -0.033 & 0.855 & -0.070 & 0.706 & -0.036 & 0.842 & & \\
\hline$S T D D L Y$ & & & & & & & & & & & -1.692 & 0.008 \\
\hline$\mu_{2}$ & 1.402 & 0.000 & 1.345 & 0.000 & 1.223 & 0.000 & 1.418 & 0.000 & 1.351 & 0.000 & 1.720 & 0.000 \\
\hline$\mu_{3}$ & 2.053 & 0.000 & 1.952 & 0.000 & 1.759 & 0.000 & 2.065 & 0.000 & 1.956 & 0.000 & 2.405 & 0.000 \\
\hline$\mu_{4}$ & 3.171 & 0.000 & 3.018 & 0.000 & 2.746 & 0.000 & 3.178 & 0.000 & 3.019 & 0.000 & 3.502 & 0.000 \\
\hline Log likelihood & \multicolumn{2}{|c|}{-38.636} & \multicolumn{2}{|c|}{-39.821} & \multicolumn{2}{|c|}{-42.204} & \multicolumn{2}{|c|}{-38.564} & \multicolumn{2}{|c|}{-39.801} & \multicolumn{2}{|c|}{-36.017} \\
\hline $\mathrm{R}^{2}$ & \multicolumn{2}{|c|}{0.228} & \multicolumn{2}{|c|}{0.157} & \multicolumn{2}{|c|}{0.001} & \multicolumn{2}{|c|}{0.232} & \multicolumn{2}{|c|}{0.158} & \multicolumn{2}{|c|}{0.370} \\
\hline Observations & \multicolumn{2}{|c|}{29} & \multicolumn{2}{|c|}{29} & \multicolumn{2}{|c|}{29} & \multicolumn{2}{|c|}{29} & \multicolumn{2}{|c|}{29} & \multicolumn{2}{|c|}{29} \\
\hline
\end{tabular}

Note: $\mu_{2}$ to $\mu_{4}$ are estimates of boundary values, where the smallest boundary $\mu_{0}$ is set to -infinity, the largest boundary $\mu_{5}$ is set to infinity. $\mu_{1}$ is normalized to zero, for estimating the constant. Note that the estimated coefficient is not the marginal effect. 
Table 10. Expected sign of correlation between the degree of delay effect and the degree of procrastination $\delta_{H}$ and $\delta_{L}$

\begin{tabular}{|c|c|c|c|c|}
\hline & & sophisticates & naïfs & \\
\hline $\begin{array}{c}\text { degree of } \\
\text { delay effect }\end{array}$ & correlation & sign of correlation & correlation & sign of correlation \\
\hline high & $\delta_{H}^{S}$ & - or 0 or + & $\delta_{H}^{N}$ & + \\
\hline low & $\delta{ }_{L}^{S}$ & 0 or + & $\delta_{L}^{N}$ & 0 or + \\
\hline
\end{tabular}

Note: $\delta$ represents the correlation between the degree of delay effect and the degree of procrastination.

Superscripts $S$ and $N$ stand for sophisticates and naïfs, respectively.

Subscripts $H$ and $L$ means high degree and low degree of procrastination, respectively.

Comparison of the correlation between high and low

\begin{tabular}{lll}
\hline type of person & sophisticates & naïfs \\
& $\delta_{H}^{S} \leq \delta_{L}^{S}$ & $\delta_{H}^{N}=\delta_{L}^{N}$ \\
\hline
\end{tabular}

Comparison of the correlation between sophisticates and naïfs

\begin{tabular}{ccc}
\hline degree of delay effect & high & low \\
& $\delta_{H}^{S}<\delta_{H}^{N}$ & $\delta_{L}^{S} \leq \delta_{L}^{N}$ \\
\hline
\end{tabular}


Table 11. Procrastinating behavior of Naïfs and Sophisticates

\begin{tabular}{|c|c|c|c|c|c|c|}
\hline & $\begin{array}{l}\text { Estimated } \\
\text { coefficient }\end{array}$ & P-value & $\begin{array}{l}\text { Estimated } \\
\text { coefficient }\end{array}$ & P-value & $\begin{array}{l}\text { Estimated } \\
\text { coefficient }\end{array}$ & P-value \\
\hline Constant & 1.628 & 0.000 & 1.694 & 0.000 & 1.717 & 0.000 \\
\hline$A V T D$ & 0.828 & 0.022 & 0.963 & 0.010 & 0.944 & 0.012 \\
\hline$H D L Y$ & -0.330 & 0.247 & -0.505 & 0.090 & -0.597 & 0.057 \\
\hline$L D L Y$ & 0.220 & 0.402 & 0.294 & 0.231 & 0.308 & 0.198 \\
\hline$\mu_{2}$ & 1.409 & 0.000 & 1.535 & 0.000 & 1.600 & 0.000 \\
\hline$\mu_{3}$ & 2.016 & 0.000 & 2.168 & 0.000 & 2.248 & 0.000 \\
\hline$\mu_{4}$ & 3.130 & 0.000 & 3.282 & 0.000 & 3.363 & 0.000 \\
\hline Log likelihood & \multicolumn{2}{|c|}{-38.848} & \multicolumn{2}{|c|}{-37.554} & \multicolumn{2}{|c|}{-36.947} \\
\hline $\mathrm{R}^{2}$ & \multicolumn{2}{|c|}{0.215} & \multicolumn{2}{|c|}{0.289} & \multicolumn{2}{|c|}{0.322} \\
\hline Number of observations & \multicolumn{2}{|c|}{29} & \multicolumn{2}{|c|}{29} & \multicolumn{2}{|c|}{29} \\
\hline $\begin{array}{c}\text { Critical value of } D L Y \\
\text { (High group, Low group) }\end{array}$ & \multicolumn{2}{|c|}{$(12,17)$} & \multicolumn{2}{|c|}{$(6,23)$} & \multicolumn{2}{|c|}{$(4,25)$} \\
\hline & $\begin{array}{l}\text { Estimated } \\
\text { coefficient }\end{array}$ & P-value & $\begin{array}{l}\text { Estimated } \\
\text { coefficient }\end{array}$ & P-value & & \\
\hline Constant & 3.493 & 0.023 & 2.026 & 0.001 & & \\
\hline AVSTDTD & 2.138 & 0.024 & 1.434 & 0.004 & & \\
\hline HSTDDLY & -4.242 & 0.027 & -2.375 & 0.021 & & \\
\hline LSTDDLY & 0.836 & 0.101 & -0.749 & 0.551 & & \\
\hline$\mu_{2}$ & 2.801 & 0.034 & 1.791 & 0.000 & & \\
\hline$\mu_{3}$ & 3.413 & 0.012 & 2.461 & 0.000 & & \\
\hline$\mu_{4}$ & 5.129 & 0.002 & 3.560 & 0.000 & & \\
\hline Log likelihood & \multicolumn{2}{|c|}{-13.294} & \multicolumn{2}{|c|}{-35.637} & & \\
\hline $\mathrm{R}^{2}$ & \multicolumn{2}{|c|}{0.723} & \multicolumn{2}{|c|}{0.390} & & \\
\hline Number of observations & \multicolumn{2}{|c|}{29} & \multicolumn{2}{|c|}{29} & & \\
\hline Critical value of STDDLY & \multicolumn{2}{|c|}{0.0} & \multicolumn{2}{|c|}{0.1} & & \\
\hline (High group, Low group) & \multicolumn{2}{|c|}{$(14,15)$} & \multicolumn{2}{|c|}{$(11,18)$} & & \\
\hline
\end{tabular}

Note: Refer to the footnote of Table 9. 
Figure 1. Histogram of the degree of the delay effect, DLY

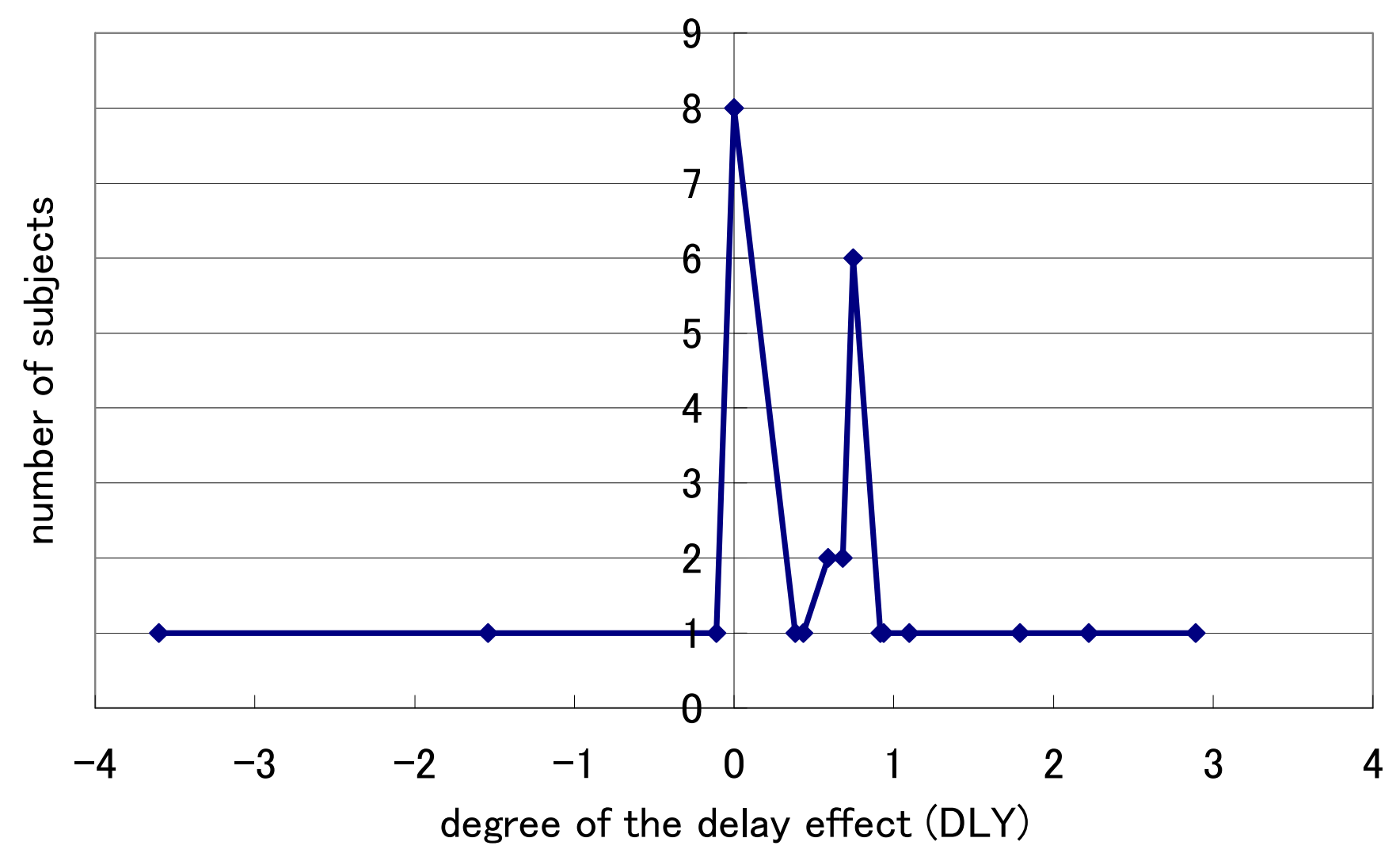


Figure 2. Histogram of the standardized degree of the delay effect, STDDLY

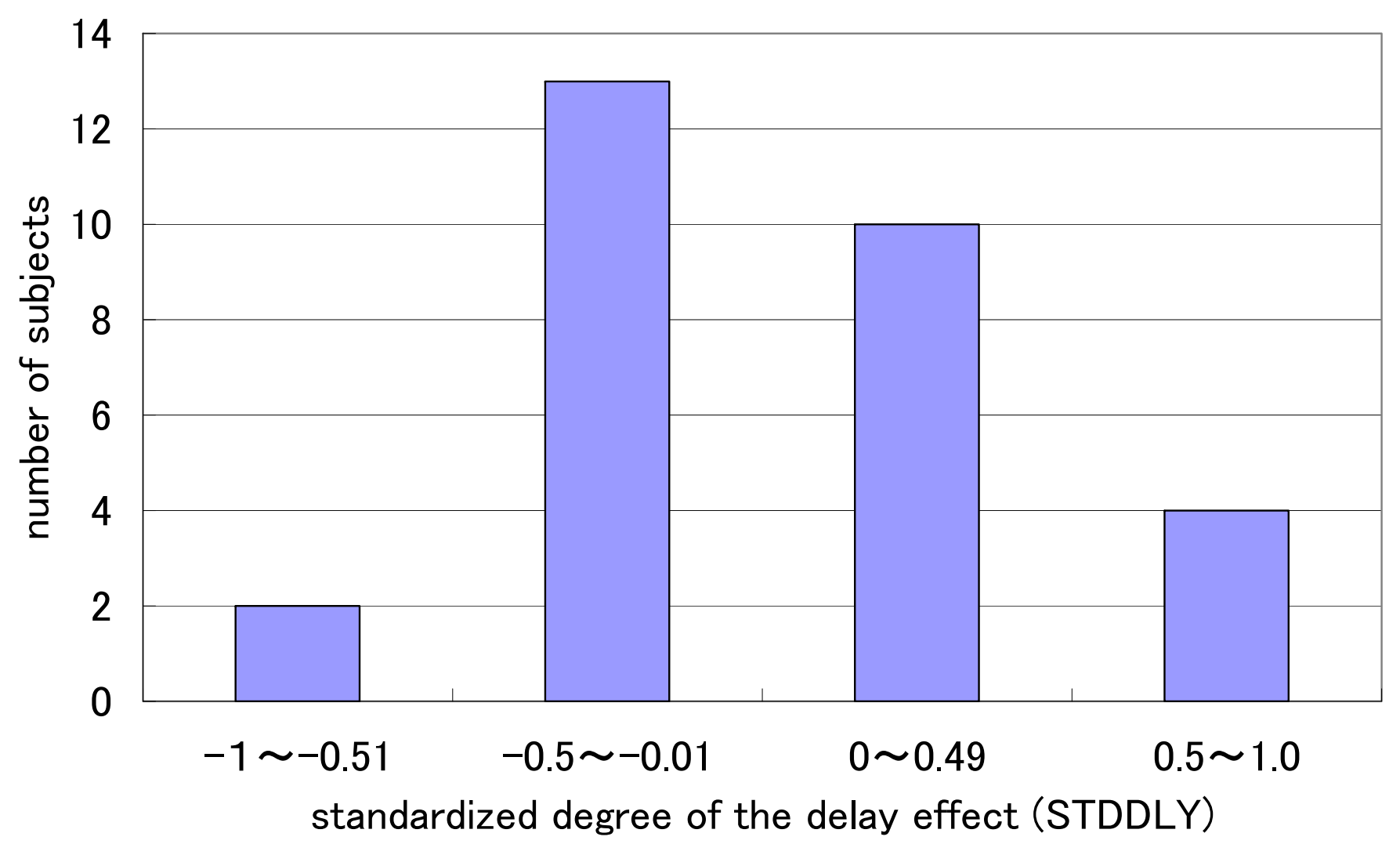




\section{Appendix: Payoff table used in the experiment (Round 1)}

(1) Payoff table (2,800.00 yuan, 1 month vs. 4 months, reward is not actually paid)

\begin{tabular}{|c|c|c|c|c|c|}
\hline $\begin{array}{l}\text { Number of the } \\
\text { option pair }\end{array}$ & $\begin{array}{l}\text { Option A (yuan) } \\
\text { (receipt at } 1 \\
\text { month later) }\end{array}$ & $\begin{array}{c}\text { Option B } \\
\quad \text { (yuan) } \\
\text { (receipt at } 4 \\
\text { month later) }\end{array}$ & $\begin{array}{c}\text { Rate of return } \\
\text { (annual rate) }\end{array}$ & \multicolumn{2}{|c|}{ Answers } \\
\hline 1 & $2,800.00$ & $2,730.00$ & $-10 \%$ & $A$ & $B$ \\
\hline 2 & $2,800.00$ & $2,765.04$ & $-5 \%$ & A & $B$ \\
\hline 3 & $2,800.00$ & $2,772.00$ & $-4 \%$ & A & $B$ \\
\hline 4 & $2,800.00$ & $2,779.04$ & $-3 \%$ & A & $B$ \\
\hline 5 & $2,800.00$ & $2,786.00$ & $-2 \%$ & A & $B$ \\
\hline 6 & $2,800.00$ & $2,793.04$ & $-1 \%$ & A & $\mathrm{B}$ \\
\hline 7 & $2,800.00$ & $2,800.00$ & $0 \%$ & A & $\mathrm{B}$ \\
\hline 8 & $2,800.00$ & $2,807.04$ & $1 \%$ & A & $B$ \\
\hline 9 & $2,800.00$ & $2,814.00$ & $2 \%$ & A & $\mathrm{B}$ \\
\hline 10 & $2,800.00$ & $2,821.04$ & $3 \%$ & A & $B$ \\
\hline 11 & $2,800.00$ & $2,828.00$ & $4 \%$ & A & $B$ \\
\hline 12 & $2,800.00$ & $2,835.04$ & $5 \%$ & A & $\mathrm{B}$ \\
\hline 13 & $2,800.00$ & $2,842.00$ & $6 \%$ & A & $\mathrm{B}$ \\
\hline 14 & $2,800.00$ & $2,849.04$ & $7 \%$ & $A$ & $B$ \\
\hline 15 & $2,800.00$ & $2,856.00$ & $8 \%$ & A & $\mathrm{B}$ \\
\hline 16 & $2,800.00$ & $2,863.04$ & $9 \%$ & A & $B$ \\
\hline 17 & $2,800.00$ & $2,870.00$ & $10 \%$ & A & $B$ \\
\hline 18 & $2,800.00$ & $2,877.04$ & $11 \%$ & A & $\mathrm{B}$ \\
\hline 19 & $2,800.00$ & $2,884.00$ & $12 \%$ & $\mathrm{~A}$ & $\mathrm{~B}$ \\
\hline 20 & $2,800.00$ & $2,891.04$ & $13 \%$ & $A$ & $B$ \\
\hline 21 & $2,800.00$ & $2,898.00$ & $14 \%$ & $A$ & $B$ \\
\hline 22 & $2,800.00$ & $2,905.04$ & $15 \%$ & A & $B$ \\
\hline 23 & $2,800.00$ & $2,912.00$ & $16 \%$ & $A$ & $B$ \\
\hline 24 & $2,800.00$ & $2,919.04$ & $17 \%$ & $A$ & $B$ \\
\hline 25 & $2,800.00$ & $2,926.00$ & $18 \%$ & $\mathrm{~A}$ & $\mathrm{~B}$ \\
\hline 26 & $2,800.00$ & $2,933.04$ & $19 \%$ & $\mathrm{~A}$ & $\mathrm{~B}$ \\
\hline 27 & $2,800.00$ & $2,940.00$ & $20 \%$ & $A$ & $B$ \\
\hline 28 & $2,800.00$ & $2,975.04$ & $25 \%$ & $A$ & $B$ \\
\hline 29 & $2,800.00$ & $3,010.00$ & $30 \%$ & A & $\mathrm{B}$ \\
\hline 30 & $2,800.00$ & $3,080.00$ & $40 \%$ & A & $\mathrm{B}$ \\
\hline 31 & $2,800.00$ & $3,150.00$ & $50 \%$ & A & $\mathrm{B}$ \\
\hline 32 & $2,800.00$ & $3,220.00$ & $60 \%$ & $A$ & $B$ \\
\hline
\end{tabular}

Notes: 1. Please circle A or B, whichever you prefer.

2. If you circled a wrong option, correct the answer with a double line. 